\title{
Cost-effectiveness of ribociclib plus endocrine therapy versus placebo plus endocrine therapy in HR-positive, HER2-negative breast cancer
}

\author{
Vivian Le, PharmD Candidate; Lixian Zhong, PhD; Nihal Narsipur, PharmD; Elizabeth Hays, PharmD Candidate; \\ Daniel Khuong Tran, PharmD Candidate; Kimberly Rosario, PharmD Candidate; and Leslie Wilson, PhD
}

\section{What is already known about this subject}

- The American Society of Clinical Oncology currently recommends endocrine therapy plus or minus a selective cyclin-dependent kinase (CDK) $4 / 6$ inhibitor as the first-line treatment for hormone receptor (HR)positive breast cancer.

- Ribociclib, a selective CDK4/6 inhibitor, plus endocrine therapy has demonstrated significant improvements in progression-free and overall survival for patients living with HR-positive breast cancer in recent clinical trials.

- Gains in response and overall survival come with trade-offs such as adverse events and costs, which are important considerations for providers and third-party payer systems when determining the value of new treatments and therapies.

\section{ABSTRACT}

BACKGROUND: The 2015 American Society of Clinical Oncology guidelines recommend first-line treatment of hormone receptor (HR)-positive breast cancer with endocrine therapy plus or minus palbociclib, a selective cyclin-dependent kinase (CDK)4/6 inhibitor. In 2018, the U.S. Food and Drug Administration approved ribociclib, a new orally available selective CDK4/6 inhibitor. While gains in progression-free survival (PFS) and overall survival (OS) from ribociclib are important for

\section{What this study adds}

- This study evaluates the costeffectiveness of ribociclib plus endocrine therapy over endocrine therapy alone in endocrine treatmentnaive, pre- and perimenopausal women living with HR-positive, HER2-negative advanced breast cancer.

- This analysis determines and evaluates important factors affecting the cost-effectiveness of ribociclib plus endocrine therapy using sensitivity analyses.

- Results of this study provide additional information for consideration by payers, clinicians, and formulary decision makers in determining the benefit of adding ribociclib to therapy regimens. clinical and treatment outcomes, trade-offs in adverse events (AEs) and additional costs necessitate cost-effectiveness analysis (CEA) to assist consideration by third-party payer systems, physicians, and patients.

OBJECTIVES: To (a) develop a Markov model and (b) determine the cost-effectiveness of ribociclib plus endocrine therapy versus endocrine therapy alone as treatment for premenopausal and perimenopausal patients with HR-positive, human epidermal growth factor receptor 2 (HER2)-negative breast cancer.

\section{Author affiliations}

Vivian Le, PharmD Candidate; Nihal Narsipur, PharmD; Elizabeth Hays, PharmD Candidate; Daniel Khuong Tran, PharmD Candidate; Kimberly Rosario, PharmD Candidate; and Leslie Wilson, PhD, Department of Clinical Pharmacy, University of California, San Francisco. Lixian Zhong, PhD, Texas A\&M College of Pharmacy, Kingsville.

\section{AUTHOR CORRESPONDENCE:}

Leslie Wilson, 415.990.1012;

leslie.wilson@ucsf.edu

J Manag Care Spec Pharm 2021;27(3):327-38

Copyright $(2021$, Academy of Managed Care Pharmacy. All rights reserved.

METHODS: A lifetime 3-state Markov model ("stable," "progressed," and "dead" health states) was developed using a U.S. payer perspective. Transition probabilities were calculated based on OS and PFS outcomes from the randomized controlled phase 3 trial MONALEESA-7. These Kaplan-Meier curves were extended to lifetime by estimating best-fit distributions using loglogistic distribution for ribociclib curves and Weibull distribution for placebo curves. Costs were obtained from national data sources using 2019 U.S. dollars (USD) and discounted by $3 \%$. Utilities were obtained via published 
breast cancer literature and were included for each health state and for time spent with each AE. Results were expressed as an incremental cost-effectiveness ratio (ICER) expressed as USD per quality-adjusted life-year (QALY) saved. Treatments were assumed to be cost-effective based on a willingness-to-pay (WTP) threshold of $\$ 150,000$ per QALY gained. Base-case, 1-way sensitivity tornado diagrams and probabilistic sensitivity analyses demonstrated changes in the ICER and were driven by the cost of ribociclib and the utility of remaining in the stable health state.

RESULTS: Ribociclib plus endocrine therapy was cost-effective at an ICER of $\$ 124,513$ per QALY when compared with endocrine therapy alone at a WTP threshold of $\$ 150,000$. The ribociclib plus endocrine therapy arm had an effectiveness of 5.28 QALYs and a total cost of $\$ 385,112$, while placebo plus endocrine therapy provided only 2.46 QALYs at a lower total cost of $\$ 67.246$. The model was sensitive to the cost of ribociclib and the utility of time spent in the stable health state. Probabilistic sensitivity analysis demonstrated that endocrine therapy alone was cost-effective until a WTP of $\$ 125,000$ and was cost-effective $72 \%$ of the time at the WTP threshold.

CONCLUSIONS: Ribociclib plus endocrine therapy is more cost-effective than endocrine therapy alone. Professionals in managed care settings should consider the pharmacoeconomic benefits of ribociclib for the treatment of HR-positive, HER2-negative breast cancer as they make value-based formulary decisions. Further CEAs should be considered as direct treatment comparison trials between CDK4/6 inhibitors are completed in the future.

In 2020, an estimated 279,100 new cases of breast cancer and 42,690 deaths in women living in the United States were reported by the American Cancer Society. ${ }^{1}$ Biological pathways by which breast cancer may metastasize can be inferred by the presence of hormone receptor (HR) and human epidermal growth factor receptor 2 (HER2). In HR-positive, HER2-negative advanced breast cancer particularly, cyclin-dependent kinases (CDK)4/6 are known to promote continued progression and growth of the cancer. ${ }^{2}$ Current American Society of Clinical Oncology (ASCO) guidelines recommend first-line treatment of HR-positive breast cancer with aromatase inhibitors plus or minus palbociclib, a selective CDK4/6 inhibitor. ${ }^{3}$ Second-line therapy includes an alternative endocrine therapy agent, fulvestrant, plus or minus palbociclib. Chemotherapy is not recommended in initial HR-positive breast cancer treatment except in immediately life-threatening disease due to higher toxicity and lower quality of life (QOL) versus endocrine therapy. ${ }^{3}$ Since the release of ASCO guidelines in 2015, 2 new orally available selective CDK4/ 6 inhibitors were approved by the U.S. Food and Drug Administration (FDA) in 2018, ribociclib (KISQALI) and abemaciclib (VERSENIO). While head-to-head clinical trials are still needed between the 3 , indirect meta-analysis has shown equal effectiveness among the CDK $4 / 6$ inhibitors, making ribociclib and abemaciclib equal alternatives to palbociclib for first-line treatment regimens. ${ }^{4,5}$

Recent clinical trials of ribociclib have demonstrated that ribociclib combined with endocrine therapy results in significantly longer overall and progression-free survival (PFS) over endocrine therapy alone. The MONALEESA-7 (Mammary Oncology Assessment of LEE011's [Ribociclib] Efficacy and Safety-7) trial was the first to study PFS and overall survival (OS) of ribociclib plus endocrine therapy in endocrine treatment-naive premenopausal and perimenopausal patients. ${ }^{6-14}$ These patients tend to be underrepresented in clinical trials of breast cancer despite differences in molecular alterations of key breast cancer driver genes, tumor-suppressor genes, and genes involved in signaling pathways. ${ }^{15}$ MONALEESA-7 showed a significantly longer PFS and OS survival with ribociclib plus endocrine therapy than with endocrine therapy alone among these patients. ${ }^{14}$ While gains in PFS and OS are important for clinical and treatment outcomes, it is essential to examine the trade-offs for patients in adverse events (AE) and additional costs to the health care system. These factors are important considerations for third-party payers when determining overall value of new therapies and for providers and patients determining treatment effect on their QOL and willingness to pay (WTP).

Our objective was to determine the cost-effectiveness of ribociclib plus endocrine therapy versus endocrine therapy alone as treatment for premenopausal and perimenopausal patients with HR-positive, HER2-negative breast cancer from a U.S. payer's perspective. This study will help health care decision makers to better understand ribociclib with respect to its efficacy, toxicity, and cost, and to make informed value-based decisions when evaluating the benefit of adding ribociclib to a treatment regimen or formulary.

\section{Methods}

We performed a cost-effectiveness analysis (CEA) of ribociclib plus endocrine therapy versus placebo plus endocrine therapy. The methods in conducting this CEA adhere to the recommendations of good research practices for model transparency and validation from the International Society for Pharmacoeconomics and Outcomes Research (ISPOR), the Society of Medical Decision Making, and the Consolidated Health Economic Evaluation Reporting Standards statement from the ISPOR Task Force. ${ }^{16-18}$

We used a lifetime 3-state Markov model with stable, progressed, and dead health states. Transition probabilities were based on OS and PFS outcomes from the randomized 
controlled phase 3 trial MONALEESA-7.. ${ }^{14}$ We included utilities of each health state, as well as time spent with each AE. The study was done from a payer's perspective and included direct medical costs associated with treatment. Costs of drug, treatment of AEs, and subsequent drug treatments were obtained from national literature using 2019 U.S. dollars (USD) and discounted by $3 \%$. CEA results were expressed as an incremental cost-effectiveness ratio (ICER) expressed as USD per life-year (LY) saved and qualityadjusted life-year (QALY) saved. Treatments were assumed to be cost-effective based on a WTP threshold of $\$ 150,000$ per QALY gained. ${ }^{16,19}$

\section{MARKOV MODEL}

Eligible patients were women aged between 18 and 59 years; premenopausal or perimenopausal at time of trial entry with histologically or cytologically confirmed HR-positive, HER2-negative advanced breast cancer; and no previous endocrine therapy or more than 1 previous line of chemotherapy.

The 3-state Markov model was developed with 2 choice branches at the decision node to reflect MONALEESA-7 treatment options: ribociclib plus endocrine therapy or endocrine therapy alone (placebo; Figure 1). Both treatment groups received goserelin (at a dose of $3.6 \mathrm{mg}$, administered subcutaneously on day 1 of each 28-day cycle) and either nonsteroidal aromatase inhibitor (letrozole $2.5 \mathrm{mg}$ or anastrozole $1 \mathrm{mg}$ ) or tamoxifen (20 mg) orally once daily. Each cycle length was designated as 1 month, or 28 days, to match treatment protocol. From the decision nodes, all patients began in the "stable" health state and then either stayed stable or transitioned to the "progressed" or "dead" health states. Patients who transitioned to the progressed state could stay progressed or enter the dead state but could not go back to the stable state. "Death" was the absorbing state (Supplementary Figure 1, available in online article). Probability of progression and mortality after progression were obtained from the fitted OS and PFS curves in the MONALEESA-7 trial (see Survival Curve Model Fitting section). Patients could also die directly from the stable state from background mortality estimated from 2017 life tables. $^{20}$ The simulation continued until 360 months, when nearly all patients $(94.7 \%$ in ribociclib arm and $99.9 \%$ in placebo arm) had transitioned to the dead terminal node.

Our base-case analysis included utilities of each disease state and disutilities of AEs to assess effectiveness as QALYs. Further analyses were performed to create an LYs model by assessing the model without any utilities, which was expressed as cost per LY to determine the effect of utility estimates on our results. The Markov model, 1-way sensitivity analyses, tornado diagrams, and probabilistic

\section{\begin{tabular}{l|l} 
FIGURE 1 Study Markov Model \\
\hline
\end{tabular}}

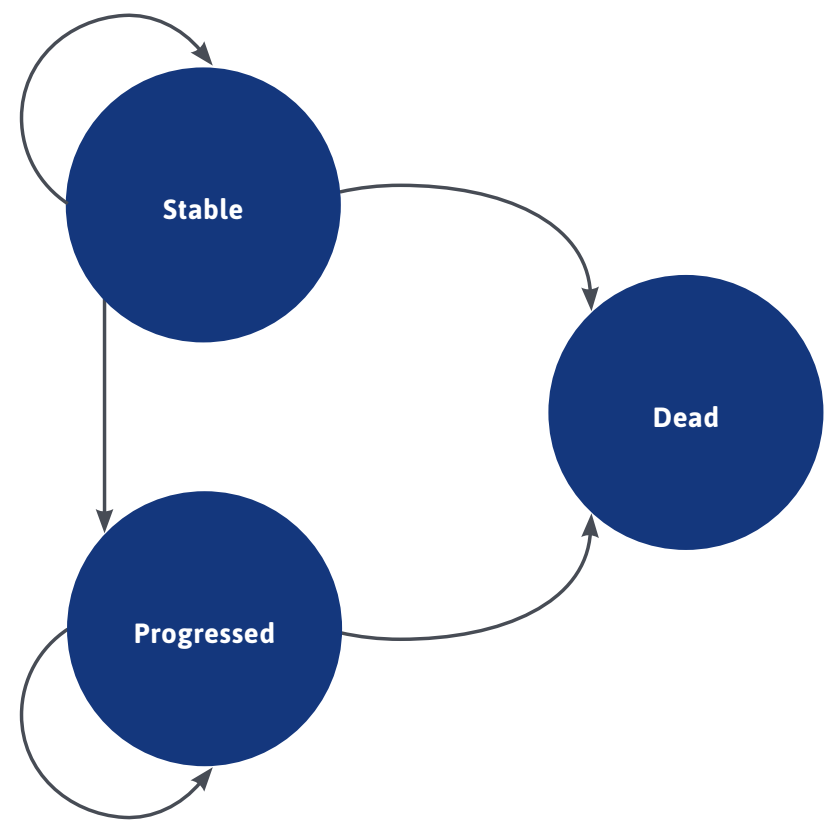

Note: This figure shows a schematic representation of the Markov model with disease states and transitions between each state. In the stable state, ribociclib plus endocrine therapy or placebo plus endocrine therapy for first-line treatment of HR-positive, HER2-negative advanced breast cancer in pre-and perimenopausal patients. Once transitioned to the progressed state, switched to a subsequent therapy: chemotherapy (paclitaxel), hormonal therapy (fulvestrant), or palliative care.

$H E R 2$ = human epidermal growth factor receptor 2; HR=hormone receptor.

sensitivity analyses were performed using TreeAge software (TreeAge, Williamstown, MA).

\section{SURVIVAL CURVE MODEL FITTING}

Transition probabilities represented the proportion of patients who moved from 1 health state into another health state after 1 cycle of treatment. ${ }^{19}$ To calculate transition probabilities, PFS and OS data for both treatment arms were extracted from published Kaplan-Meier curves in MONALEESA-7 using a validated graphical digitizer (Engauge Digitizer version 12.1, Torrance, CA). ${ }^{14}$ To extend the OS and PFS curves to lifetime, we extracted these Kaplan-Meier curves using maximum likelihood estimation estimates from Hoyle and Henley (2011) using RStudio (RStudio, Boston, MA) to determine the best-fitting lifetime OS and PFS probabilities by using the lowest Akaike information criterion and Bayesian information criterion. ${ }^{21}$ Best-fit lifetime distribution was loglogistic for the ribociclib curves and Weibull distribution for the placebo curves. 


\section{FIGURE 2 Projected Overall and Progression-Free Survival Curves Using Data Extrapolated from MONALEESA-7 for 360 Months}

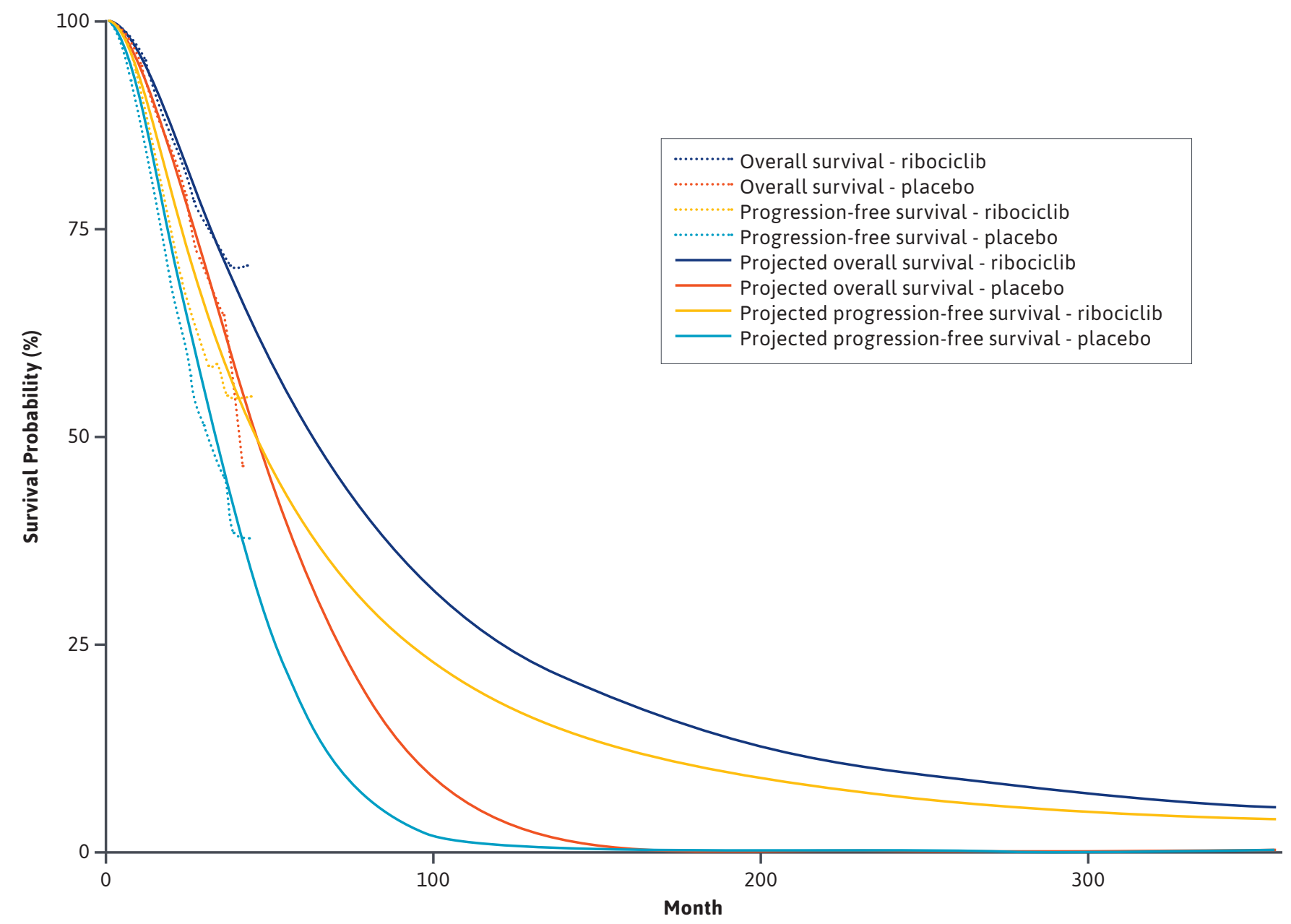

Note: Projected curves for the ribociclib arm followed loglogistic distributions, while projected curves for the placebo arm followed Weibull distributions in accordance with best-fit distributions from Kaplan-Meier curves. Patients in the ribociclib treatment arm experienced higher rates of overall survival and progression-free survival.

These distributions were used to project lifetime survival curves that were then used to calculate transitional probabilities for each monthly cycle in our Markov model (Figure 2).

\section{COSTS AND UTILITIES}

Costs were calculated for each arm to include monthly drug therapy, management of AEs, and other treatment-associated costs (Table1). Average wholesale price of drug therapies were taken from RED BOOK Online via Micromedex and discounted by $16 \%$ to account for discounts and rebates patients received from drug manufacturers. ${ }^{22}$ Any weightbased dosing was calculated based on an average weight of $77 \mathrm{~kg}$ for a healthy woman and rounded up to $80 \mathrm{~kg}$ due to an assumed $2.7 \mathrm{~kg}$ weight gain while in treatment for breast cancer. ${ }^{23,24}$ Other costs, including guideline-based management of AEs, were estimated using Current Procedural Terminology codes and the 2019 Clinical Laboratory Fee Schedule from Centers for Medicare \& Medicaid Services for lab costs, Health Care Cost and Utilization Project for hospitalization costs, and the 2019 American Medical Association Healthcare Medical Fees Directory for physician costs..$^{25,26}$ 


\section{TABLE 1 Model Input Parameters, Distribution, and Range}

\begin{tabular}{|c|c|c|c|c|}
\hline \multicolumn{5}{|l|}{ Input Parameter } \\
\hline Drug Cost & Monthly Cost & Low & High & Reference Source \\
\hline $\begin{array}{l}\text { Rubociclib } 600 \mathrm{mg} \times 21 \text { days followed } \\
\text { by } 7 \text { days off }\end{array}$ & 13,349 & 10,012 & 16,687 & $22 ; \pm 25 \%$ \\
\hline $\begin{array}{l}\text { Goserelin } 3.6 \mathrm{mg} \text { on } \\
\text { day } 1 \text { of each } 28 \text {-day cycle }\end{array}$ & 640.33 & 480.25 & 800.42 & $22 ; \pm 25 \%$ \\
\hline Letrozole $2.5 \mathrm{mg}$ once daily ${ }^{\mathrm{a}}$ & 426.42 & 319.81 & 533.02 & $22 ; \pm 25 \%$ \\
\hline Anastrazole $1 \mathrm{mg}$ once daily & 312.82 & 234.61 & 391.02 & $22 ; \pm 25 \%$ \\
\hline Tamoxifen 20 mg once daily & 88.67 & 66.50 & 110.84 & $22 ; \pm 25 \%$ \\
\hline Treatment Arm Cost ${ }^{\mathrm{a}}$ & Monthly Cost & Low & High & Reference Source \\
\hline $\begin{array}{l}\text { Ribociclib plus endocrine therapy } \\
\text { (NSAID) }\end{array}$ & 14.376 & 10,782 & 17,970 & $22,25,26$; corresponding guidelines, $\pm 25 \%$ \\
\hline Placebo plus endocrine therapy & 1,024 & 768.26 & 1,280 & $22,25,26$; corresponding guidelines, $\pm 25 \%$ \\
\hline Subsequent Treatment Cost ${ }^{a}$ & Monthly Cost & $\begin{array}{l}\text { Weighted Cost } \\
\text { (Ribociclib) }\end{array}$ & $\begin{array}{l}\text { Weighted Cost } \\
\text { (Placebo) }\end{array}$ & Reference Source \\
\hline Palliative care & 2,965 & 975.34 & 874.86 & 42 \\
\hline Chemotherapy (paclitaxel) & 261.71 & 80.08 & 95.53 & $22,25,26$; corresponding guidelines, $\pm 25 \%$ \\
\hline Hormonal therapy (fulvestrant) & 904.06 & 405.02 & 387.84 & $22,25,26$; corresponding guidelines, $\pm 25 \%$ \\
\hline Cost of progression & 11,133 & - & - & 43 \\
\hline AE Cost & $\begin{array}{l}\text { Weighted Cost of } \\
\text { AE Treatment }\end{array}$ & Low & High & Reference Source \\
\hline Ribociclib plus endocrine therapy & 16,462 & 12,346 & 20,577 & $22,25,26$; corresponding guidelines, $\pm 25 \%$ \\
\hline Placebo plus endocrine therapy & 3,789 & 2,842 & 4,736 & $22,25,26$; corresponding guidelines, $\pm 25 \%$ \\
\hline Utilities for Disease States & Utilities & Low & High & Reference Source \\
\hline Stable disease, base case & 0.715 & 0.50 & 0.80 & 28,29 \\
\hline Progressed disease, base case & 0.443 & 0.41 & 0.69 & 28,29 \\
\hline Utilities for AEs & Utilities & Low & High & Reference Source \\
\hline $\begin{array}{l}\text { Neutropenia or leukopenia } \\
\text { (febrile and hospitalized) }\end{array}$ & 0.33 & 0.25 & 0.41 & $44 ; \pm 25 \%$ \\
\hline Anemia & 0.64 & 0.34 & 0.94 & 45; range calculated from SD \\
\hline Thrombocytopenia & 0.65 & 0.33 & 0.67 & 46 \\
\hline Sepsis & 0.20 & 0.15 & 0.25 & $29 ; \pm 25 \%$ \\
\hline Hepatobiliary toxicity & 0.216 & 0.16 & 0.27 & $36 ; \pm 25 \%$ \\
\hline Pulmonary toxicity & 0.461 & 0.35 & 0.58 & $47 ; \pm 25 \%$ \\
\hline QT interval prolongation & 0.60 & 0.45 & 0.75 & $48 ; \pm 25 \%$ \\
\hline Renal toxicity & 0.39 & 0.29 & 0.49 & $49 ; \pm 25 \%$ \\
\hline Pulmonary embolism & 0.395 & 0.30 & 0.49 & $50 ; \pm 25 \%$ \\
\hline
\end{tabular}

Note: Costs are represented in U.S. dollars.

${ }^{a}$ Costs include all treatment-associated costs, including outpatient initial visit, follow-up visits, lab draws, monitoring, follow-up imaging, and pretreatment regimens as applicable and recommended by treatment guidelines.

$A E=$ adverse event; $C M S=$ Centers for Medicare \& Medicaid Services; HCUP=Healthcare Cost and Utilization Project; NSAID=nonsteroidal anti-inflammatory drug; $S D=$ standard deviation 
TABLE 2 Summary of Cost-Effectiveness of Ribociclib plus Endocrine Therapy and Placebo plus Endocrine Therapy as First-Line Treatment of HR-Positive, HER2-Negative Advanced Breast Cancer in Pre- and Perimenopausal Patients

\begin{tabular}{|c|c|c|c|c|c|}
\hline Treatment & $\begin{array}{l}\text { Total Cost } \\
(95 \% \mathrm{Cl})\end{array}$ & $\begin{array}{c}\text { Total } \\
\text { Effectiveness }\end{array}$ & $\begin{array}{c}\text { Incremental } \\
\text { Cost }\end{array}$ & $\begin{array}{c}\text { Incremental } \\
\text { Effect }\end{array}$ & $\begin{array}{l}\text { Annual } \\
\text { ICER }\end{array}$ \\
\hline QALY Model & & QALY & & QALY & \$/QALY \\
\hline Placebo plus endocrine therapy & 67,246 & 2.83 & - & - & - \\
\hline Ribociclib plus endocrine therapy & 385,112 & 5.28 & 317,866 & 2.46 & 129,299 \\
\hline Life-Years Model & & LY & & LY & \$/LY \\
\hline Placebo plus endocrine therapy & 67,246 & 4.44 & - & - & - \\
\hline Ribociclib plus endocrine therapy & 385,112 & 8.07 & 317,866 & 3.63 & 87,473 \\
\hline
\end{tabular}

Note: Costs are represented in U.S. dollars.

$\mathrm{Cl}=$ confidence interval; ICER = incremental cost-effectiveness ratio; $L Y=$ life-year; $Q A L Y=$ quality-adjusted life-year.

All costs were adjusted to 2019 USD using the Consumer Price Index, and all costs were discounted at an annual rate of $3 \%$ to account for time preference of costs.

Ribociclib cost was determined using the dosing regimen of $600 \mathrm{mg}$ administered orally once daily for $21 \mathrm{consecu-}$ tive days, followed by 7 days off, for a complete cycle of 28 days. Patients were assumed to be on treatment of ribociclib or placebo until disease progression. Patients who progressed during MONALEESA-7 were switched to a subsequent therapy, including chemotherapy, hormonal therapy, other therapy, or a combination of the 3. Our model presumed these therapies to be paclitaxel, fulvestrant, and palliative care, respectively, based on clinical guidelines.,27 The cost of these subsequent therapies was weighted according to the percentage of patients receiving each subsequent therapy in MONALEESA-7. Thus, patients in the progressed state were switched from the cost of original treatment to the cost of new subsequent therapy, as done in MONALEESA-7.

Costs for grade 3/4 AE management, determined by treatment guidelines and clinical expert assessment for each $\mathrm{AE}$, were calculated to include treatment medications, diagnostic lab tests, monitoring lab tests, follow-up outpatient visits, and hospitalization costs for managing the AEs. Costs were calculated for each individual $\mathrm{AE}$ and weighted according to the percentage of patients experiencing each AE. Notable AEs included hematological (neutropenia, anemia, and thrombocytopenia) and nonhematological (hepatobiliary toxicity, prolonged QT interval, pulmonary toxicity, renal toxicity, and sepsis; Table 1). ${ }^{9,14}$ Grade $1 / 2$ AEs were not included due to minimal medical services required and limited cost impact; data available also did not allow for differentiation of grade 2 from grade 1 events. Discontinuation, dose reduction, and delay of therapy due to toxicity were not included due to lack of available data. Utilities for stable and progressed disease states and disutility for grade $3 / 4 \mathrm{AEs}$ in patients with breast cancer were found from literature estimates (Table 1). ${ }^{28,29}$ Both a disease state utility and a weighted disutility for each $\mathrm{AE}$ were included.

\section{SENSITIVITY ANALYSES}

Deterministic 1-way sensitivity and probabilistic sensitivity analyses (PSA) were performed using TreeAge software with varied parameters to account for uncertainty in all parameters. Deterministic sensitivity analyses varied cost parameters by a $\pm 25 \%$ plausible range around mean estimates, while utility parameters used plausible ranges reported in literature (Table 1). ${ }^{28}$ For PSA, cost estimates were assigned gamma distributions, giving it infinity at the upper end and bounding it to zero at the lower end, to account for the high variability of cost. Disutility and utility estimates were assigned beta distributions, bounding it by 1 at the upper end and 0 at the lower end, while transition probabilities were assigned PERT distributions. ${ }^{19}$ PSA was performed for 10,000 Monte Carlo simulations.

\section{Results}

\section{BASE-CASE COST-EFFECTIVENESS ANALYSIS}

We found that ribociclib plus endocrine therapy compared with endocrine therapy alone was cost-effective at an ICER of $\$ 124,513$ per QALY, which is less than our WTP threshold of $\$ 150,000$ per QALY. The ribociclib arm cost $\$ 369,862$ while the placebo arm was least costly at $\$ 63,760$. Ribociclib had an additional life expectancy of 5.28 QALYs 


\section{FIGURE 3 WTP Acceptability Curve from Probabilistic Sensitivity Analysis}

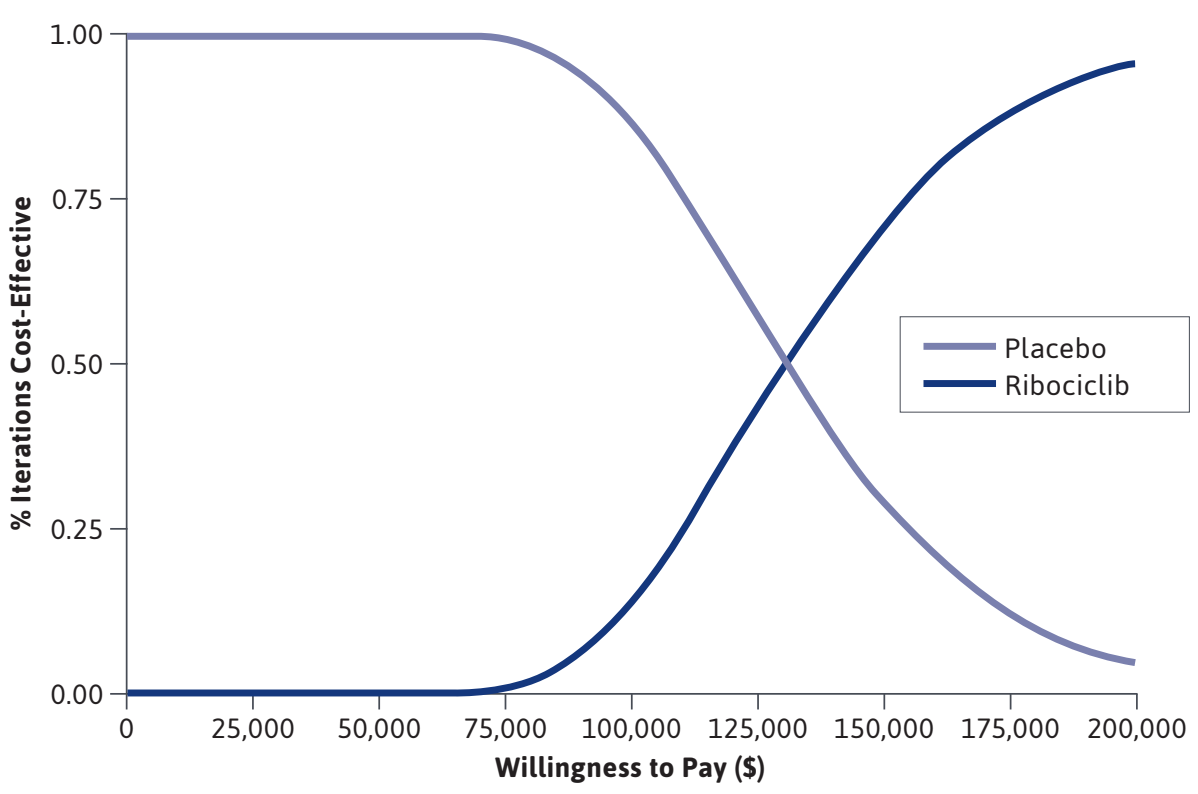

Note: Ribociclib was not a viable option until a WTP threshold of $\$ 54,000 / Q A L Y$ and was cost-effective for $71.7 \%$ of Monte Carlo simulations at a WTP of $\$ 150,000$. All costs are represented in U.S. dollars. $\mathrm{QALY}=$ quality-adjusted life-year; $W T P=$ willingness to pay.

while placebo life expectancy was 2.46 QALYs shorter (2.86 QALYs). When utilities were excluded from our model, the ribociclib arm was associated with 8 LYs as compared with only 4.4 LYs for the placebo arm, leading to an ICER of $\$ 84,236$ per LY saved. In the life-year model, ribociclib plus endocrine therapy provided an additional $3.63 \mathrm{LYs}$ with an incremental cost of $\$ 306,102$ as compared with placebo plus endocrine therapy (Table 2).

\section{SENSITIVITY ANALYSES}

To determine which variables were most influential, 1-way sensitivity and threshold analyses were performed on all variables within the base-case QALYs model and LYs model excluding utilities. Given plausible ranges, only 2 variables affected ribociclib costeffectiveness: ribociclib cost (mean $\$ 14,376 /$ month) and utility of stable disease (0.715). The tornado diagram shows that these 2 variables alone accounted for $98.2 \%$ of the variability in our model (Supplementary Figure $\underline{2}$, available in online article). A $\pm 25 \%$ change in cost of ribociclib led to a $\pm 26.4 \%$ change in the base-case ICER; ribociclib was still cost-effective up to a monthly cost of $\$ 16,600$ (15.5\% higher). The LYs model indicated that ribociclib was cost-effective up to an even higher monthly cost of $\$ 24,150$ (68.0\% higher).

Increasing the utility of the stable disease state from 0.715 to a high input of 0.8 from reported literature decreased the ICER by $10.0 \%$, as patients experienced higher utility and effectiveness when surviving longer on ribociclib; decreasing the utility from 0.715 to a low input of 0.5 increased the ICER by $39.2 \% .^{28}$ Threshold analyses indicated that ribociclib remained cost-effective until a utility of 0.61 for the stable disease state (14.7\% lower). A utility of 0.61 is a possible utility for patients experiencing stable advanced breast cancer, though lower on the plausible range. ${ }^{28}$ Unlike the stable state, utility of the progressed state was not as effective, with no change in cost-effectiveness when changed in a plausible range from 0.41 to $0.69{ }^{28}$ Despite common and notable AEs, including neutropenia and sepsis, cost and disutility of AEs did not cause a remarkable effect on the ICER. Utility of both stable and progressed disease states and cost of progression and treatment were significantly more effective than cost or disutility of AEs. No other disutility or utility value besides stable disease state utility caused the ICER to become not costeffective, likely due to the extended amount of time the ribociclib group spent in the stable state compared with the placebo group.

The acceptability curve (Figure 3 ) indicated that treatment with ribociclib does not become cost-effective over endocrine therapy alone until a WTP threshold of $\$ 54,000$ per QALY is reached. Given a WTP of $\$ 150,000$, ribociclib was cost-effective for $71.7 \%$ of all iterations, indicating strong robustness in our results. By a WTP threshold of $\$ 200,000$, ribociclib was cost-effective for $95.5 \%$ of all iterations. Thus, with increasing acceptable WTP thresholds for cancer therapies, ribociclib plus endocrine therapy became more cost-effective, while endocrine therapy alone became less cost-effective.

\section{Discussion}

Based on our CEA and a WTP threshold of $\$ 150,000$ per QALY, ribociclib plus endocrine therapy is cost-effective when compared with placebo plus endocrine therapy for first-line 
treatment of HR-positive, HER2-negative advanced breast cancer in pre- or perimenopausal patients, with an ICER of $\$ 124,513$ per QALY. Some analysts indicate that there should be variation across disease types, especially for oncology drugs, and a WTP threshold of $\$ 200,000-\$ 300,000$ per QALY would be more reasonable due to increases in health care spending over time and subsequent health gains. ${ }^{5} \mathrm{We}$ preferred a more conservative and widely accepted estimate of $\$ 150,000$ per QALY. If a WTP of $\$ 100,000$ is used, then the cost of ribociclib would have to be reduced by $21.5 \%$ to approximately $\$ 11,300$ per cycle in order for ribociclib to remain cost-effective.

Previous studies have suggested that cost-effectiveness seemed unlikely due to high cost. Niraula (2018) calculated that ribociclib would cost $\$ 1.92$ million to prevent 1 PFS event, causing potential financial toxicity. ${ }^{30}$ This high cost is partly due to the long exposure to ribociclib (23.8 months) before PFS events, due to its effectiveness. ${ }^{10}$ Previous studies have also found ribociclib plus letrozole cost-effective versus letrozole alone in postmenopausal women despite the \$1.76 million indicated to prevent 1 PFS. ${ }^{30,31}$ Niraula also argued that the addition of CDK4/6 inhibitors could cause financial toxicity due to lack of OS improvements and require infinite cost to prevent 1 death. ${ }^{30}$ However, ribociclib has since shown significant improvements in OS, and our results indicate that ribociclib can still be cost-effective despite its high cost burden..$^{14}$ Nonetheless, the incredible cost burden must be acknowledged considering that it is an optional addition to first-line treatment with endocrine therapy.

For cost variables, 1-way sensitivity and tornado analyses indicated that the ICER was most sensitive to the total drug cost of ribociclib. Ribociclib plus endocrine therapy is naturally more expensive than endocrine therapy alone due to inclusion of ribociclib, extensive monitoring of lab results, and treatment guidelines surrounding ribociclib. ${ }^{9}$ Given $\$ 211.89$ per $200 \mathrm{mg}$ tablet, a 28 -day course of treatment with ribociclib, (21 days of oral $600 \mathrm{mg}$ ribociclib plus 7 days off) amounted to approximately $\$ 13,349$ per month for drugs alone. ${ }^{32}$ This amount was added to endocrine therapy (goserelin plus tamoxifen, anastrozole, or letrozole), which ranged from $\$ 729.00-\$ 1,267$ per month. Thus, endocrine therapy with ribociclib cost 10.5 times more than the most expensive endocrine therapy (letrozole plus goserelin). Treatment-associated costs were an additional \$701.28 per month without accounting for follow-up outpatient visits. Due to the significant monthly cost of ribociclib, the ICER was heavily influenced by the total drug cost. However, threshold analysis indicated that the monthly drug cost could increase up to $23.1 \%$ higher and still be cost-effective.
As the availability of generic versions and rebates occur, ribociclib will continue to be more cost-effective.

Our model could not include costs of discontinuation and dose reduction from toxicity due to lack of available data from the clinical trial. Dose reduction, in particular, raises concerns for drug wastage since alternative CDK4/6 inhibitors like palbociclib have significant drug wastage costs of $\$ 1,124$ per patient per year from dose changes. This limitation is not applicable to ribociclib since prior studies indicated that dose reductions in ribociclib resulted in $\$ 0$ drug wastage since unused tablets could be used in future treatment cycles. ${ }^{33}$ In fact, for postmenopausal patients in MONALEESA-2, a dose reduction in ribociclib resulted in decreased costs per month of PFS gained. ${ }^{34}$ Similar reductions in cost for pre- and perimenopausal patients requiring dose adjustments could also be expected due to the reusability of unused tablets.

For utility, the model potentially underestimated the effect of progressed state utility since tornado analyses were only run from 0.41 to 0.69 , while base value was 0.443 due to ranges given in literature. ${ }^{28}$ However, even when progressed utility was reduced to 0 , ribociclib remained cost-effective, indicating that it was not effective in our model. This is attributable to patients spending most of their time in the stable state. Once progressed, patients quickly proceeded to the dead state. Patients in the progressed state never exceeded $12.8 \%$ in the ribociclib group or $19.4 \%$ in the placebo group in any 1 given cycle.

In contrast, patients in both arms spent the most time in the stable state, and the most significant difference was that the ribociclib group had more patients remaining stable. In the ribociclib arm, $56.6 \%$ of patients remained stable versus $41.2 \%$ patients in the placebo arm by month 40 , while the percentage of patients in the progressed state was more equivalent at $12.3 \%$ versus $16.4 \%$, respectively. By month $150,13.6 \%$ of patients remained stable in the ribociclib group versus only $0.1 \%$ patients remained stable in the placebo group, indicating that patients in the ribociclib group remained stable for significantly longer. Thus, MONALEESA-7's PFS rate and difference between stable groups were a larger driving force of ribociclib's effectiveness than that for patients who had progressed.

There are concerns that MONALEESA-7's results are not generalizable since it was a multinational trial that showed most favorable results in Asia, weakly beneficial results in North America, and only $3 \%$ of its participants were black. ${ }^{10}$ This weaker benefit in non-Asian patients has also been reflected in previous clinical trials such as MONALEESA-2. ${ }^{11}$ Thus, we recognize that non-Asian patients may not see utility and, consequently, cost-effectiveness results as strongly favorable as those described in this CEA. However, 
we expect to see some favorable results due to the robust results and general benefit seen in non-Asian patients. Future studies can benefit from research published after the completion of our analysis, which compared the QOL of each treatment group over time within the MONALEESA-7 trial using the Quality of Life Questionnaire Core 30, a health-related QOL measure. ${ }^{35}$ Although these results cannot be used directly in a CEA study because they are not utilities, their results can be mapped to the EuroQol-5 Dimension to obtain utilities within a CEA. Although our utilities are reflective of the QOL advantages of treatment with ribociclib plus endocrine therapy, and we would not expect a different CEA decision, future studies should map and use their detailed QOL assessments.

Compared with utility of disease states, disutility of AEs played a lesser role in ribociclib's cost-effectiveness. Although ribociclib yielded remarkable improvements in OS, we expected treatment AEs to be a significant burden for patients. In addition to neutropenia at $63.5 \%, 4.8 \%$ of participants experienced sepsis in the ribociclib arm compared with $2.4 \%$ in the placebo arm, which had a utility of 0.2 in patients with breast cancer. ${ }^{14,29}$ Grade $3 / 4$ hepatobiliary toxicity was experienced in $11 \%$ of patients treated with ribociclib versus $6.8 \%$ treated with placebo, which had a utility of $0.216 .14,36$ These numbers indicated that patients were likely to experience substantially decreased utility due to both the prevalence and severity of these AEs. However, sensitivity analyses showed that both cost and disutility of AE had little effect on the ICER. This could be because patients only experienced AEs for brief periods of time (i.e., 4 days for neutropenia), and $\mathrm{AE}$ detriments were significantly outweighed by improvements in survival and prolongation of stable disease states.

Adherence is typically assumed to be near $100 \%$ in a randomized control trial (RCT) setting unless stated otherwise. However, a study on real-world clinical and economic outcomes of palbociclib indicated growing evidence of suboptimal effectiveness of oral anticancer medications (OAMs) due to significant low patient adherence, adverse effect profiles, and sociodemographic factors. ${ }^{37}$ Although the study evaluated palbociclib, these findings can be applied equally to other CKD4/6 inhibitors. Adherence issues due to toxicity or other factors can lead to lower survival rates, increased risk of recurrence, and higher health care costs. Psychosocial factors, such as depression, side effect concerns, and race and geographical differences, also have significant influences on patient adherence to OAMs. ${ }^{38}$ Thus, medication management is highly important for patients on OAMs due to narrow therapeutic ranges and safety margins associated with these drugs. ${ }^{37}$ Given these findings, it is reasonable to assume that adherence issues outside of an RCT setting would lower the costeffectiveness of ribociclib if its adherence is lower than that of the control group.

While ribociclib has 2 competitors, palbociclib and abemaciclib, our study did not include direct comparisons since there are no comparable clinical trials among the 3 treatments. While both competitors had clinical trials conducted in pre- and perimenopausal women (PALOMA-3 and MONARCH-2), they studied previous heavily treated women, requiring their endocrine therapy to be fulvestrant unlike MONALEESA-7.39,40 Thus, the studies did not have equivalent background treatments suitable even for indirect comparisons across these trials. ${ }^{4,41}$ Future comparable studies on the use of palbociclib/abemaciclib plus aromatase inhibitors in pre- or perimenopausal women are needed before determining which CDK4/6 inhibitor is most cost-effective.

\section{LIMITATIONS}

Our model has limitations worth mentioning, since it required several assumptions based on limitations of the clinical trial (MONALEESA-7). First, when denoting utilities, each patient had a chance to experience all AEs once, regardless of length of drug treatment. This could be an underestimation of $\mathrm{AE}$ frequency since some events could occur more than once, but there was no certainty in whether these events were allowed to occur more than once in MONALEESA-7 before treatment discontinuation. We estimated the cost of each AE treatment based on the expected time in each disease state. For example, neutropenia was estimated to receive treatment for 4 days only. Therefore, we believe we did a fair estimation of AE costs and utilities, given the available data.

Second, MONALEESA-7 did not specify time of drug treatment discontinuations for reasons other than progression, making it difficult to determine if such discontinuations occurred early in treatment for some or most patients or if this was at a constant rate. Since discontinuation rate for reasons other than progression only amounted to $12.9 \%-14.2 \%$ of total discontinuations, these possible earlier discontinuations were not accounted for in the model. Moreover, when patients did discontinue treatment due to progression, assumptions were made on their subsequent therapy based on guidelines rather than exact data from the clinical trial patient experience, which was not available. We also assumed patients receiving "other therapy" received palliative care due to the nature of advanced cancer. ${ }^{14}$

Despite these weaknesses, our model was robust to most variables in our sensitivity analysis. Additionally, our probabilistic sensitivity analysis showed strong stability 
of the cost-effectiveness of ribociclib within our WTP, demonstrating it was cost-effective $71.7 \%$ of the time.

\section{Conclusions}

This CEA demonstrates that ribociclib plus endocrine therapy is more cost-effective compared with endocrine therapy alone. This analysis provides important value-based information for indication-specific pricing of treatments and is useful for clinicians and managed care specialists considering whether to add ribociclib to endocrine therapy or formulary. Endocrine therapy alone is still a first-line treatment according to ASCO guidelines, and decision makers may be deterred from adding ribociclib when considering the additional cost and significant AEs. ${ }^{3}$

The results of this CEA lend some support for the addition of ribociclib to conventional endocrine therapies for pre- and perimenopausal women. Ribociclib's cost-effectiveness is mainly influenced by current drug costs and our assumptions about utility of stable disease while on drug treatment. Although the cost of ribociclib and its competitors may change in the future due to further competition and potential FDA approval of newer CDK4/6 inhibitors, this study can support decision makers in the meantime.

\section{DISCLOSURES}

No outside funding supported this study. The authors have nothing to disclose.

\section{REFERENCES}

1. American Cancer Society. Cancer facts \& figures 2020. June 2020. Accessed February 4, 2021. https://www.cancer.org/content/dam/cancer-org/ research/cancer-facts-and-statistics/ annual-cancer-facts-and-figures/2020/ cancer-facts-and-figures-2020.pdf
2. Shapiro GI. Cyclin-dependent kinase pathways as targets for cancer treatment. J Clin Oncol. 2006;24(11):1770-83. doi: 10.1200/JCO.2005.03.7689

3. Rugo HS, Rumble RB, Macrae E, et al. Endocrine therapy for hormone receptor-positive metastatic breast cancer: American Society of Clinical Oncology guideline. J Clin Oncol. 2016;34(25):3069103. doi: $10.1200 / J C O .2016 .67 .1487$

4. Petrelli F, Ghidini A, Pedersini R, et al. Comparative efficacy of palbociclib, ribociclib and abemaciclib for ER+ metastatic breast cancer: an adjusted indirect analysis of randomized controlled trials. Breast Cancer Res Treat. 2019;174(3):597-604. doi: 10.1007/s10549-019-05133-y

5. Neumann PJ, Cohen JT, Weinstein MC. Updating cost-effectiveness-the curious resilience of the $\$ 50,000$-per-QALY threshold. N Engl J Med. 2014;371(9):79697. doi: 10.1056/NEJMp1405158

6. Cardoso F, Senkus E, Costa A, et al. 4th ESO-ESMO International Consensus Guidelines for Advanced Breast Cancer (ABC 4)†. Ann Oncol. 2018;29(8):1634-57. doi: 10.1093/annonc/mdy192

7. National Comprehensive Cancer Network. Breast cancer. (version 4.2020). Accessed February 4, 2021. https://www. nccn.org/professionals/physician_gls/ default.aspx\#breast

8. Slamon DJ, Neven P, Chia S, et al. Phase III randomized study of ribociclib and fulvestrant in hormone receptorpositive, human epidermal growth factor receptor 2-negative advanced breast cancer: MONALEESA-3. J Clin Oncol. 2018;36(24):2465-72. doi: 10.1200/ JCO.2018.78.9909

9. Kisqali (ribociclib) tablets, for oral use. Novartis Pharmaceuticals Corporation. July 2020. Accessed February 4, 2021. https://www.novartis.us/sites/www. novartis.us/files/kisqali.pdf

10. Tripathy D, Im SA, Colleoni M, et al. Ribociclib plus endocrine therapy for premenopausal women with hormonereceptor-positive, advanced breast cancer (MONALEESA-7): a randomised phase 3 trial. Lancet Oncol. 2018;19(7):904-15. doi: 10.1016/S1470-2045(18)30292-4
11. Hortobagyi GN, Stemmer SM, Burris HA, et al. Updated results from MONALEESA-2, a phase III trial of firstline ribociclib plus letrozole versus placebo plus letrozole in hormone receptor-positive, HER2-negative advanced breast cancer. Ann Oncol. 2018;29(7):154147. doi: 10.1093/annonc/mdy155

12. Hortobagyi GN, Stemmer SM, Burris HA, et al. Ribociclib as first-line therapy for HR-positive, advanced breast cancer. N Engl J Med. 2016;375(18):1738-48. doi: 10.1056/NEJMoa1609709

13. Tripathy D, Bardia A, Sellers WR. Ribociclib (LEE011): mechanism of action and clinical impact of this selective cyclin-dependent kinase 4/6 inhibitor in various solid tumors. Clin Cancer Res. 2017;23(13):3251-62. doi: 10.1158/1078-0432. CCR-16-3157

14. Im SA, Lu YS, Bardia A, et al. Overall survival with ribociclib plus endocrine therapy in breast cancer. N Engl J Med. 2019;381(4):307-16. doi: 10.1056/ NEJMoa1903765

15. Bellet M, Gray KP, Francis PA, et al. Twelve-month estrogen levels in premenopausal women with hormone receptor-positive breast cancer receiving adjuvant triptorelin plus exemestane or tamoxifen in the suppression of ovarian function trial (SOFT): the SOFT-EST substudy. J Clin Oncol. 2016;34(14):1584-93. doi: 10.1200/JCO.2015.61.2259

16. Gold, MR, Siegel, JE, Russell, LB, Weinstein, MC. Cost-Effectiveness in Health and Medicine. New York: Oxford University Press; 1996.

17. Eddy DM, Hollingworth W, Caro JJ, et al. Model transparency and validation: a report of the ISPOR-SMDM Modeling Good Research Practices Task Force-7. Value Health. 2012;15(6):843-50. doi: 10.1016/j.jval.2012.04.012

18. Husereau D, Drummond M, Petrou S, et al. Consolidated Health Economic Evaluation Reporting Standards (CHEERS)-explanation and elaboration: a report of the ISPOR Health Economic Evaluation Publication Guidelines Good Reporting Practices Task Force. Value Health. 2013;16(2):231-50. doi: 10.1016/j. jval.2013.02.002 
19. Briggs, AH, Claxton, K, Sculpher, MJ. Decision Modelling for Health Economic Evaluation. New York: Oxford University Press; 2006.

20. Social Security. Actuarial life table. 2017. Accessed February 4, 2021. https:// www.ssa.gov/oact/STATS/table4c6.html

21. Hoyle MW, Henley W. Improved curve fits to summary survival data: application to economic evaluation of health technologies. BMC Med Res Methodol. 2011;11:139. doi: 10.1186/1471-2288-11-139

22. Congressional Budget Office.

Prescription drug pricing in the private sector. January 2007. Accessed February 4, 2021. https://www.cbo.gov/sites/ default/files/110th-congress-2007-2008/ reports/01-03-prescriptiondrug.pdf

23. Centers for Disease Control and Prevention. National Center for Health Statistics. Body measurements. January 14, 2021. Accessed February 4, 2021. https:// www.cdc.gov/nchs/fastats/body-measurements.htm

24. van den Berg MMGA, Winkels RM, de Kruif JThCM, et al. Weight change during chemotherapy in breast cancer patients: a meta-analysis. BMC Cancer. 2017;17(1):259. doi: 10.1186/s12885-017-3242-4

25. Centers for Medicare \& Medicaid Services. Clinical laboratory fee schedule files. CY 2019 Q4 release: revised for October 2019. File 19CLABQ4. Accessed February 4, 2021. https://www.cms. gov/Medicare/Medicare-Fee-forService-Payment/ClinicalLabFeeSched/ Clinical-Laboratory-Fee-Schedule-Files

26. Agency for Healthcare Research and Quality. Healthcare Cost and Utilization Project (HCUP). Free health care statistics. 2006-2009. Accessed February 4, 2021. http://hcupnet.ahrq.gov/

27. Partridge AH, Rumble RB, Carey LA, et al. Chemotherapy and targeted therapy for women with human epidermal growth factor receptor 2-negative (or unknown) advanced breast cancer: American Society of Clinical Oncology Clinical Practice Guideline. J Clin Oncol. 2014;32(29):3307-29.
28. Lloyd A, Nafees B, Narewska J, Dewilde S, Watkins J. Health state utilities for metastatic breast cancer. $\mathrm{Br} \mathrm{J}$ Cancer. 2006;95(6):683-90. doi: 10.1038/ sj.bjc. 6603326

29. Earle CC, Chapman RH, Baker CS, et al. Systematic overview of costutility assessments in oncology. J Clin Oncol. 2000;18(18):3302-17. doi: 10.1200/ JCO.2000.18.18.3302

30. Niraula S. Why upfront use of CDK inhibitors for the treatment of advanced breast cancer may be wasteful, and how we can increase their value. Breast. 2018;43:81-84.

31. Mistry R, May JR, Suri G, et al. Costeffectiveness of ribociclib plus letrozole versus palbociclib plus letrozole and letrozole monotherapy in the first-line treatment of postmenopausal women with HR+/HER2- advanced or metastatic breast cancer: a U.S. payer perspective. J Manag Care Spec Pharm. 2018;24(6):51423. doi: 10.18553/jmcp.2018.24.6.514

32. IBM. Micromedex RED BOOK Online. Active ingredient: Kisqali. Database. 2015. Accessed February 4, 2021. https://www. ibm.com/products/micromedex-red-book

33. Biskupiak, J, Oderda, G, Brixner, et al. Quantification of economic impact of drug wastage in oral oncology medications: comparison of 3 methods using palbociclib and ribociclib in advanced or metastatic breast cancer. J Manag Care Spec Pharm. 2019;25(8):859-66. doi: 10.18553/jmcp.2019.25.8.859

34. Giuliani J, Bonetti A. The introduction of a third CDK $4 / 6$ inhibitor does not change the cost-effectiveness profile in first and subsequent-lines after progression or relapse during previous endocrine therapy in patients with hormone receptor positive $(\mathrm{HR}+) /$ human epidermal receptor-2 negative (HER-2) advanced or metastatic breast cancer. J Oncol Pharm Pract. 2020;26(6):1486-91. doi: 10.1177/1078155220934531826-831
35. Harbeck N, Franke F, VillanuevaVazquez R, et al. Health-related quality of life in premenopausal women with hormone-receptor-positive, HER2negative advanced breast cancer treated with ribociclib plus endocrine therapy: results from a phase III randomized clinical trial (MONALEESA-7). Ther Adv Med Oncol. 2020;12:1758835920943065. doi: $10.1177 / 1758835920943065$

36. Kabeshova A, Ben Hariz S, Tsakeu E, Benamouzig R, Launois R. Costeffectiveness analysis of rifaximin- $\alpha$ administration for the reduction of episodes of overt hepatic encephalopathy in recurrence compared with standard treatment in France. Therap Adv Gastroenterol. 2016;9(4):473-82. doi: 10.1177/1756283X16644249

37. Babcock A, Ali AA, Balkrishnan R, et al. Real-world clinical and economic outcomes associated with palbociclib for HR-positive/HER2 negative metastatic breast cancer: a commentary. J Manag Care Spec Pharm. 2020;26(7):826-31. doi: 10.18553/jmcp.2020.26.7.826

38. Lin C, Clark R, Tu P, Bosworth HB, Zullig LL. Breast cancer oral anti-cancer medication adherence: a systematic review of psychosocial motivators and barriers. Breast Cancer Res Treat. 2017;165(2):247-60.

39. Loibl S, Turner NC, Ro J, et al. Palbociclib combined with fulvestrant in premenopausal women with advanced breast cancer and prior progression on endocrine therapy: PALOMA-3 results. Oncologist. 2017;22(9):1028-38. doi: 10.1634/theoncologist.2017-0072

40. Sledge GW Jr, Toi M, Neven P, et al. The effect of abemaciclib plus fulvestrant on overall survival in hormone receptorpositive, ERBB2-negative breast cancer that progressed on endocrine therapyMONARCH 2: a randomized clinical trial. JAMA Oncol. 2019;6(1):116-24. doi: 10.1001/ jamaoncol.2019.4782 
41. Dodwell D, Vergote I. A comparison of fulvestrant and the third-generation aromatase inhibitors in the second-line treatment of postmenopausal women with advanced breast cancer. Cancer Treat Rev. 2005;31(4):274-82. doi: 10.1016/j. ctrv.2005.03.009

42. Sorensen SV, Goh JW, Pan F, et al. Incidence-based cost-of-illness model for metastatic breast cancer in the United States. Int J Technol Assess Health Care. 2012;28(1):12-21. doi: 10.1017/ S026646231100064X

43. Reyes C, Engel-Nitz NM, DaCosta Byfield S, et al. Cost of disease progression in patients with metastatic breast, lung, and colorectal cancer. Oncologist. 2019;24(9):1209-18. doi: 10.1634/theoncologist.2018-0018
44. Lee EK, Wong WW, Trudeau ME, Chan KK. Cost-effectiveness of prophylactic granulocyte colony-stimulating factor for febrile neutropenia in breast cancer patients receiving FEC-D. Breast Cancer Res Treat. 2015;150(1):169-80. doi: 10.1007/s10549-015-3309-3

45. Martin SC, Gagnon DD, Zhang L, Bokemeyer C, Van Marwijk Kooy M, van Hout B. Cost-utility analysis of survival with epoetin-alfa versus placebo in stage IV breast cancer. Pharmacoeconomics. 2003;21(16):1153-69. doi: 10.2165/00019053-200321160-00002

46. Handorf EA, McElligott S, Vachani A, et al. Cost effectiveness of personalized therapy for first-line treatment of stage IV and recurrent incurable adenocarcinoma of the lung. J Oncol Pract. 2012;8(5):267-74. doi: 10.1200/JOP.2011.000502
47. Doyle S, Lloyd A, Walker M. Health state utility scores in advanced nonsmall cell lung cancer. Lung Cancer. 2008;62(3):374-80. doi: 10.1016/j.lungcan. 2008.03.019

48. Hall PS, Hulme C, McCabe C, Oluboyede Y, Round J, Cameron DA. Updated cost-effectiveness analysis of trastuzumab for early breast cancer: a UK perspective considering duration of benefit, long-term toxicity and pattern of recurrence. Pharmacoeconomics. 2011;29(5):415-32. doi: 10.2165/11588340-000000000-00000

49. Todd C. Chemotherapy-related toxicities and costs signal place for amifostine. Pharmacoecon Outcomes News. 1997;122(1):3-5. doi:10.1007/BF03271822

50. Shih V, Chan A, Xie F, Ko Y. Health state utility assessment for breast cancer. Value Health Reg Issues. 2012;1(1):93-97. doi: 10.1016/j.vhri.2012.03.009 